Error-Related Brain Activity as a Biomarker for Cocaine Relapse

Addiction is characterized by the observation that substance-dependent individuals continue to use substances despite the negative consequences, such as social, interpersonal, or physical problems. The ability to adequately monitor negative consequences in behavior, referred to as error processing, is necessary for optimal behavioral performance to guide one's behavior toward one's long-term goals (eg, maintain abstinence from substance use). It has been previously reported that cocaine-dependent individuals show a decreased sensitivity to errors and this has been attributed to reduced activation in the anterior cingulate cortex (ACC; Kaufman et al, 2003). Likewise, electrophysiological research has shown that the errorrelated negativity (ERN)-which represents the brain's automatic detection of an error-is reduced in cocaine users compared with healthy controls (Franken et al, 2007). It has been theorized that a reduced ERN is a representation of the notion that errors are perceived as less meaningful or motivationally relevant in substance-dependent individuals (Hajcak, 2012). This may underlie their persistence of drug taking despite the clear adverse consequences. Additionally, it is conceivable that these brain dysfunctions associated with error processing also have a role in drug relapse.

Error processing is typically measured using reaction time tasks with high chances to make errors, such as the Go-Nogo task or Eriksen flanker task. To examine the predictive role of error processing in drug relapse, we measured event-related potentials (ERPs) in response to an Eriksen flanker task in cocaine-dependent patients during their first week of detoxification treatment (Marhe et al, 2013). In this task, letter strings are presented and participants are required to respond as quickly and accurately as possible to a target letter. This task is frequently used to measure error processing, as participants easily make mistakes on this task. First, the results confirmed that the ERN amplitude was indeed reduced in cocaine-dependent patients as compared with non-dependent controls. Most interestingly, ERN amplitude predicted cocaine use after treatment, over and above other relevant predictors measured at baseline such as substance use severity and subjective cocaine craving. A reduced ERN at baseline was associated with a higher number of days of cocaine use at 3-month follow-up. Another recent study using functional magnetic resonance imaging found that the reduced error-related brain activity in areas such as the dorsal ACC, thalamus, and insula is associated with cocaine relapse after treatment (Luo et al, 2013). The results of both these studies indicate that cocaine-dependent patients exhibiting underactive error-related brain activity are more at risk of relapse.

Error-related brain activity might serve as a biomarker helping to identify patients vulnerable for relapse already early in treatment. Although there is knowledge on the underlying brain processes of error processing (Kaufman et al, 2003; Luo et al, 2013), further investigation of the underlying neural circuitry and neurochemistry using neuroimaging and (combined) pharmacological approaches will further advance this research area. Regarding the results of Marhe et al (2013), it could be beneficial in the future to routinely assess the ERN amplitude in cocaine-dependent patients at the start of detoxification treatment. The idea to use electroencephalography (EEG) as a screening instrument has gained interest, specifically for ERP components that have good psychometric properties, such as the ERN (Hajcak, 2012; Hoffmann and Falkenstein, 2012). In addition, EEG is a noninvasive, relative inexpensive, and accessible biomarker. Therefore, it would be very feasible to use this measure in large-scale (genetic) studies. Future studies should reconfirm the association between the ERN and drug relapse and further examine the sensitivity and specificity of the ERN as a predictor of cocaine relapse. Ultimately, treatment programs could be tailored to the patient's need to improve outcomes.

Reshmi Marhe ${ }^{1,2}$ and Ingmar Franken ${ }^{1}$

${ }^{1}$ Institute of Psychology, Erasmus University

Rotterdam, Rotterdam, The Netherlands;

${ }^{2}$ Department of Child and Adolescent Psychiatry,

VU University Medical Center, Amsterdam,

The Netherlands

E-mail:r.marhe@debascule.com

FUNDING AND DISCLOSURE

The authors declare no conflict of interest.

Franken $1 \mathrm{HA}$, Van Strien JW, Franzek EJ, Van de Wetering BJM (2007). Error-processing deficits in patients with cocaine dependence. Biol Psychol 75: 45-51.

Hajcak $G$ (2012). What we've learned from mistakes: Insights from error-related brain activity. Curr Dir Psychol Sci 21: 101-106.

Hoffmann S, Falkenstein M (2012). Predictive information processing in the brain: errors and response monitoring. Int J Psychophysio/ 83: 208-212.

Kaufman JN, Ross TJ, Stein EA, Garavan H (2003). Cingulate hypoactivity in cocaine users during a GO-NOGO task as revealed by event-related functional magnetic resonance imaging. J Neurosci 23: 7839-7843.

Luo X, Zhang S, Hu S, Bednarski SR, Erdman E, Farr OM (2013). Error processing and gender-shared and -specific neural predictors of relapse in cocaine dependence. Brain 136: 1231-1244.

Marhe R, Van de Wetering BJM, Franken IHA (2013). Error-related brain activity predicts cocaine use after treatment at 3-month follow-up. Biol Psychiatry 73: 782-788.

Neuropsychopharmacology Reviews (2014) 39, 241; doi: 10.1 038/npp.2013.245

\section{Parkinson's Disease Biomarkers: Resources for Discovery and Validation}

Parkinson's disease (PD) is currently diagnosed using clinical features. While experienced neurologists can typically diagnose PD with 70-90\% accuracy, there are many situations early in the course of the disease when clinical diagnosis is less precise. There is a paucity of objective measures that could be employed to improve the diagnosis, stratify patients by subtypes, and track underlying disease progression. Development of innovative new therapies that slow or stop the progression of the disease would be accelerated by such objective biomarkers. Various imaging modalities including specific dopaminergic markers and 
morphometric measures are available, but have not been firmly established as markers for these purposes. Markers that can be detected in easily obtainable biofluids would be ideal for these purposes and would increase the power to detect the effect of therapeutic agents in a shorter time with reduced cost.

Unbiased exploratory examination of panels of RNAs, both mRNA and noncoding RNAs, exosomes, proteins, antibodies, and metabolites in small individual cohorts have yielded promising candidate markers that yearn for replication in independent cohorts (Kroksveen et al., 2011). More targeted approaches to identify biomarkers based on PD pathophysiology have shown disease-related proteins as strong candidates. Alpha-synuclein is decreased in the cerebrospinal fluid of PD patients (Hong et al., 2010), although significant overlap with controls makes it less useful in assisting individual diagnosis. Other potential protein candidates include DJ-1 and inflammatory cytokines. Post-translationally modified forms of these proteins may improve the biomarker specificity. Most of these studies have utilized CSF (Parnetti et al., 2013), although increased oxidized DJ-1 in PD patients was detected in blood (Saito et al., 2009) and epidermal growth factor levels in plasma has been shown to predict cognitive decline in PD (ChenPlotkin et al., 2011). Ultimately one could predict that a panel of multiple biochemical markers could be combined to increase the accuracy of diagnosis and disease progression. Heterogeneity of patient populations and lack of standardization of collection methods may contribute to the inconsistent and variable observations in the literature.

A major need in the field is the availability of biospecimens from well-characterized cohorts for discovery of new biomarkers and validation. In response to these needs, three major programs have been launched. The Parkinson's Progression Marker Initiative (PPMI) is a prospective study of 400 newly diagnosed PD patients and 200 controls that will be followed over 5 years and collect extensive clinical (motor and non-motor information), imaging, and biosample (blood, cere- brospinal fluid, DNA/RNA from blood, urine) information from all 600 subjects at sites around the world (Marek, 2011). PPMI expanded to include a prodromal cohort of 100 individuals at risk for developing $\mathrm{PD}$ to develop biomarkers that are present prior to the onset of clinical motor symptoms. The Fox Investigation for New Discovery of Biomarkers (BioFIND) is a study supported in collaboration with National Institute of Neurological Diseases and Stroke (NINDS) focused on novel biomarker discovery by enrolling 120 rigorously defined clinically typical PD in mid-stage and 120 age- and gender-matched controls at one timepoint in US sites. BioFIND was launched to serve as a platform to test new biomarkers in somewhat narrow spectrum of clinically typical PD in moderate stages to maximize the chance of discovering differences in a less heterogeneous population. The Parkinson's Disease Biomarker Program (PDBP) is a program designed to support new and existing biomarker cohorts that collect biospecimens using standardized protocols. Biospecimens and data from all above studies are available to the research community. Through standardization and coordination of data and sample collection, we are optimistic that new markers will emerge which will assist in clinical trials and ultimately result in improved management of the disease.

\section{Mark Frasier ${ }^{1}$ and Un Jung Kang ${ }^{2}$ \\ ${ }^{1}$ The Michael J Fox Foundation for Parkinson's Research, New York, NY, USA; ${ }^{2}$ Movement Disorders Division, The Neurological Institutes of New York, Columbia University College of Physicians and \\ Surgeons, New York, NY, USA \\ E-mail: mfrasier@michaeljfox.org}

\section{FUNDING AND DISCLOSURE}

M.F. is an employee of the Michael $J$ Fox Foundation and U.J.K. is a recipient of several grants from the Michael J Fox Foundation. U.J.K. has been funded by $\mathrm{NIH}$, Michael J Fox Foundation for Parkinson Research, and American Parkinson Disease Association for research and is on the medical advisory board for Caremark/CVS

Marek K (2011). The Parkinson Progression Marker Initiative (PPMI). Prog Neurobiol 95: 629-635.

Chen-Plotkin AS, Hu WT, Siderowf A, Weintraub D, Goldmann Gross R, Hurtig HI et al (2011). Plasma epidermal growth factor levels predict cognitive decline in Parkinson disease. Ann Neurol 69: 655-663.

Hong Z, Shi M, Chung KA, Quinn JF, Peskind ER, Galasko D et al (2010). DJ-1 and alpha-synuclein in human cerebrospinal fluid as biomarkers of Parkinson's disease. Brain 133: 713-726.

Kroksveen AC, Opsahl JA, Aye TT, Ulvik RJ, Berven FS (2011). Proteomics of human cerebrospinal fluid: discovery and verification of biomarker candidates in neurodegenerative diseases using quantitative proteomics. J Proteomics 74: 371-388.

Parnetti L, Castrioto A, Chiasserini D, Persichetti E, Tambasco N, El-Agnaf O et al (2013). Cerebrospinal fluid biomarkers in Parkinson disease. Nat Rev Neurol 9: 131-140.

Saito Y, Hamakubo T, Yoshida Y, Ogawa Y, Hara Y, Fujimura $\mathrm{H}$ et al (2009). Preparation and application of monoclonal antibodies against oxidized DJ-1. Significant elevation of oxidized DJ-1 in erythrocytes of early-stage Parkinson disease patients. Neurosci Lett 465: 1-5.

Neuropsychopharmacology Reviews (2014) 39, 241-242; doi: | 0. I 038/npp.2013.246

\section{Neurotherapeutic Implications of Brain-Immune Interactions}

Results suggest a cause and effect relationship between inflammatory cytokines and symptoms relevant to a number of psychiatric illnesses including mood and anxiety disorders, as well as schizophrenia. In addition, data indicate that immune cells may have a critical role in neuronal integrity and the prevention of developmental diseases including Autism Spectrum Disorders (ASD). These findings highlight the nuanced role of the immune system in brain health and illness, and emphasize the exciting potential of neurotherapeutics that target the immune system to treat neuropsychiatric disorders.

A recent clinical trial was conducted to determine whether antagonism of the inflammatory cytokine tumor necrosis factor (TNF) would reduce depressive symptoms in patients with treatment resistant depression (TRD), thereby testing the cytokine hypothesis of depression (Raison et al, 2013). Interestingly, only TRD patients with a baseline peripheral blood concentration of C-reactive protein (CRP-a readily available biomarker of inflammation) $>5 \mathrm{mg} / \mathrm{l}$ exhibited a clinically significant response to infliximab 